\title{
Foreword
}

\section{Pediatric hydrocephalus: systematic literature review and evidence-based guidelines}

\author{
Ann Marie Flannery, M.D., ${ }^{1}$ Catherine A. Mazzola, M.D., ${ }^{2}$ \\ Paul Klimo JR., M.D., M.P.H., 3,4 \\ Ann-Christine Duhaime, M.D. ${ }^{5}$ Lissa C. Baird, M.D., ${ }^{6}$ \\ MandeEP S. TAMBer, M.D., Ph.D., \\ David D. Limbrick JR., M.D., Ph.D., ${ }^{8}$ Dimitrios C. NiKas, M.D., 9 \\ Joanna Kemp, M.D.,${ }^{1}$ Alexander F. Post, M.D.,${ }^{10}$ \\ Kurtis I. Auguste, M.D., ${ }^{11}$ Asim F. Choudhri, M.D., ${ }^{12}$ \\ Laura S. Mitchell, ${ }^{13}$ And Debby Buffa ${ }^{14}$
}

${ }^{1}$ Department of Neurological Surgery, Saint Louis University, St. Louis, Missouri; '2Division of Pediatric Neurological Surgery, Goryeb Children's Hospital, Morristown, New Jersey; ${ }^{3}$ Department of Neurosurgery, University of Tennessee Health Science Center, and ${ }^{4}$ Le Bonheur Children's Hospital, Memphis, Tennessee; ${ }^{5}$ Department of Pediatric Neurosurgery, Massachusetts General Hospital, Boston, Massachusetts; ${ }^{6}$ Department of Neurological Surgery, Oregon Health \& Science University, Portland, Oregon; ${ }^{7}$ Department of Pediatric Neurological Surgery, Children's Hospital of Pittsburgh, University of Pittsburgh, Pittsburgh, Pennsylvania; ${ }^{8}$ Division of Pediatric Neurosurgery, Department of Neurological Surgery, Washington University School of Medicine, St. Louis, Missouri; ${ }^{9}$ Department of Neurosurgery, University of Illinois at Chicago, Chicago, and Advocate Children's Hospital, Oak Lawn, Illinois; ${ }^{10}$ Division of Pediatric Neurological Surgery, Department of Neurosciences and Pediatrics, Goryeb Children's HospitalMorristown Medical Center, Morristown, New Jersey; ${ }^{11}$ Department of Neurosurgery, University of California, San Francisco, California; ${ }^{12}$ Departments of Radiology, Ophthalmology, and Neurosurgery, University of Tennessee Health Science Center, Memphis, Tennessee, and Le Bonheur Neuroscience Institute, Le Bonheur Children's Hospital, Memphis, Tennessee; ${ }^{13}$ Guidelines Department, Congress of Neurological Surgeons, Schaumburg, Illinois; and ${ }^{14}$ Hydrocephalus Association, Bethesda, Maryland

Pediatric hydrocephalus is the most common surgically correctable neurological problem in children from infancy through adolescence. Effective surgical interventions are available; however, these treatments are not without side effects and failures. The need for improvements in surgical interventions is well understood. What is not clearly understood and agreed upon is what treatment is best for hydrocephalus. That is why we have created the following systematic reviews and evidence-based guidelines.

In the articles contained in "Pediatric Hydrocephalus: Systematic Literature Review and Evidence-Based
Guidelines," the November 2014 supplement to the Journal of Neurosurgery: Pediatrics, the Pediatric Hydrocephalus Systematic Review and Evidence-Based Guidelines Task Force offers a systematic literature review of articles on the following topics related to hydrocephalus: infection prevention and treatment; the effect of valve design, ventricular catheter location, and third ventriculostomy on outcome; the use of assistive technologies such as endoscopy, computer-assisted navigation, and ultrasonography; the special challenges of the premature infant with hydrocephalus; and the size of the ventricle. Members of the Task Force read these articles thoroughly, rated the quality of evidence reported in each one, and made recommendations on treatment based on the quality of evidence provided. A description of our procedure is found in Part 1: Introduction and Methodology. ${ }^{1}$

Guidelines are created by evaluating the best available evidence and recommending the most effective options for management. In our analysis we looked at available treatments of hydrocephalus, the effects and complications of these treatments, and strategies to avoid and manage complications. In undertaking this review, we focused on treatment of hydrocephalus in infants, children, and adolescents younger than 18 years of age. We included patients with either congenital or acquired hydrocephalus, including communicating and noncommunicating hydrocephalus for which causes are undetermined.

This review was undertaken under the auspices of the Pediatric Section of the American Association of Neurological Surgeons and the Congress of Neurological Surgeons, and it was initiated and approved by the Joint Guidelines Committee of both groups.

The recommendations that we make eschew the use of expert opinion, relying strictly on information available in the literature. While developing this review, the Task Force used evidence-based methodologies and adhered to strict criteria defined by the Institute of Medicine's standards for conducting systematic reviews and clinical evidence-based guidelines, as well as to the methodologies described in the following articles.

It is the hope of all patients, their families, and their medical teams that someday hydrocephalus will be entirely preventable or curable. Until that time, we must make an effort to continue to refine and improve its treatment as well as the evaluation of the effectiveness of that treatment.

The members of the Pediatric Hydrocephalus Systematic Literature and Evidence-Based Guidelines Task Force of the American Association of Neurological Surgeons and 
the Congress of Neurological Surgeons are grateful for the efforts of Ms. Laura Mitchell and Ms. Pamela Shaw, and Drs. James Rutka, Alan Cohen, and Bruce Kaufman. The guidance, patience, and financial support of the Hydrocephalus Association, especially those of Ms. Debby Buffa and Ms. Dawn Mancuso, are also gratefully acknowledged. (http://www.thejns.org/doi/abs/10.3171/2014.8.PEDS14426)

\section{Disclaimer}

This clinical systematic review of and evidence-based guidelines for the treatment of pediatric hydrocephalus were developed by a physician volunteer task force. The articles contained therein are provided as an educational tool based on an assessment of current scientific and clinical information as well as accepted approaches to treatment. They are not intended to be a fixed protocol because some patients may require more or less treatment. Patient care and treatment should always be based on a clinician's independent medical judgment given individual clinical circumstances. The information in the guidelines reflects the current state of knowledge at the time of the project's completion. The presentations are designed to provide an accurate review of the subject matter that is covered. These guidelines are disseminated with the understanding that the recommendations of the authors and consultants who have collaborated in their development are not meant to replace individualized care and treatment advice from patients' physicians. If medical advice or assistance is required, the services of a competent physician should be sought.

The proposals contained in these guidelines may not be suitable for use in all circumstances. The choice to implement any particular recommendation contained in these guidelines must be made by a managing physician in light of each patient's particular situation and on the basis of existing resources.

\section{Reference}

1. Flannery AM, Mitchell L: Pediatric hydrocephalus: systematic literature review and evidence-based guidelines. Part 1: Introduction and methodology. J Neurosurg Pediatr 14 (Suppl):3-7, 2014

Please include this information when citing this paper: DOI: 10.3171/2014.8.PEDS14426. 